\title{
An Alteration in Internodal Myelin Membrane Structure in Large Sciatic Nerve Fibres in Rats with Acute Streptozotocin Diabetes and Impaired Nerve Conduction Velocity
}

\author{
M. Fukuma, J.-L. Carpentier, L. Orci, D. A. Greene, and A. I. Winegrad \\ Institut d'Histologie et d'Embryologie, Ecole de Médecine, Université de Genève, Genève, Switzerland, \\ and George S. Cox Medical Research Institute, University of Pennsylvania, Philadelphia, Pennsylvania, USA
}

Summary. Replicas of the freeze-fracture surfaces of the internodal myelin membranes of large sciatic nerve fibres from normal and diabetic rats were compared by quantitative electron microscopy. The internodal myelin of the diabetics was examined 14 days after streptozotocin $(70 \mathrm{mg} / \mathrm{kg} \mathrm{IV})$ induced persistent hyperglycaemia, conditions under which sciatic motor nerve conduction velocity (MNCV) is consistently decreased by $20 \%$. The number of intramembranous particles per unit area of both the P-face and the E-face of the internodal myelin membrane was significantly decreased in the diabetics. This alteration in the structure of the internodal myelin membrane was not found in large sciatic nerve fibres from diabetic rats treated with insulin from day 3 through 14 , or from diabetic rats fed a diet containing $1 \%$ myoinositol; these are conditions under which the development of decreased sciatic $\mathrm{MNCV}$ is prevented or ameliorated. An alteration in internodal myelin structure occurs in acute streptozotocin diabetes which may explain the associated decreased sciatic MNCV.

Key words: Myelin membrane, freeze-fracture, streptozotocin diabetic rats, insulin, myoinositol, nerve conduction velocity.

Acute experimental diabetes results in decreased sciatic motor and sensory nerve conduction velocities $[1,2]$, which may provide a model for the decreased peripheral nerve conduction velocities found in newly diagnosed juvenile diabetics [3]. The development of impaired sciatic MNCV in acute streptozotocin diabetic rats can be prevented by insulin treatment that obviates hyperglycaemia [1], or by dietary myoinositol supplementation without insulin treatment and despite severe persistent hyperglycaemia [1]. Eliasson [4] reported that acute experimental diabetes results in a marked reduction in the electrical resistance of the internodal myelin of large sciatic nerve fibres and suggested that the development of "leaky" myelin might explain the decreased nerve conduction velocity. However, a morphological basis for the decreased nerve conduction velocity or the reported decrease in the electrical resistance of internodal myelin has not been firmly identified. Segmental demyelination or obvious myelin oedema has not been observed in the peripheral nerves of rats with acute experimental diabetes [5, 6]. Extensive morphometric studies by Thomas and his coworkers $[5,7]$ provided no evidence for the loss of large myelinated fibers in the peripheral nerves of rats with acute experimental diabetes; these workers [7] dispute the suggestion of Jakobsen [6] that axonal dwindling might explain the decreased nerve conduction velocity.

Freeze-fracture $[8,9]$ is a recent addition to the methods available for the study of peripheral nerve myelin; this technique splits the lipid bilayer of the myelin membrane and permits EM examination of its hydrophobic domain. The studies that form the basis of this report were concerned with a quantitative evaluation of freeze-fracture replicas of the internodal myelin of large sciatic nerve fibres in whom sciatic MNCV is known to vary in a consistent manner as the result of our previous studies on this model [1].

\section{Material and Methods}

Male, Wistar rats, initial weight 145 to $155 \mathrm{gm}$, were fed ad libitum during the 14 days prior to the examination of their sciatic nerves. The standard diet consisted of pellets composed of $18 \%(\mathrm{~W} / \mathrm{W})$ 
vitamin-free casein, $68 \%(\mathrm{~W} / \mathrm{W})$ sucrose, $10 \%(\mathrm{~W} / \mathrm{W})$ vegetable oil, $4 \%(\mathrm{~W} / \mathrm{W})$ inorganic salts, all known rat vitamin requirements, and $0.011 \%(\mathrm{~W} / \mathrm{W})$ free myoinositol (Nutritional Biochemical Corp., Cleveland, Ohio). The same supplier provided pellets prepared from the same diet, but supplemented with $1 \%$ $(\mathrm{W} / \mathrm{W})$ free myoinositol. Experimental diabetes was induced by the injection of $70 \mathrm{mg} / \mathrm{kg}$ of streptozotocin (Upjohn Company, Kalamazoo, Michigan) in $0.1 \mathrm{ml}$ of citrate buffer, $\mathrm{pH} 4.5$ $(0.10 \mathrm{~mol} / 1)$, into the tail vein after an overnight fast. A nonfasting plasma glucose concentration in excess of $300 \mathrm{mg} / \mathrm{dl}$ at both 24 and $48 \mathrm{~h}$ after the streptozotocin administration was required for the diagnosis of experimental diabetes. The group of diabetic rats treated with insulin was maintained on the standard diet and insulin administration begun on day 3 ; the regimen employed was that previously reported to obviate hyperglycaemia during at least the 6th through the 14th day, and to consistently prevent the development of impaired sciatic MNCV [1]. Briefly, the rats received twice daily subcutaneous injections of Lente insulin; plasma glucose was determined twice daily in the morning and in the late afternoon to permit adjustment of the insulin dosage which ranged from 0.10 to $0.17 \mathrm{U} / \mathrm{gram} /$ day. The aim of treatment was to prevent the plasma glucose concentration from exceeding $152 \pm 4 \mathrm{mg} / \mathrm{dl}$, which was found in previous studies to be the mean morning plasma glucose concentration in tail vein samples from normal rats of the same strain, age, sex, and initial weight fed the standard diet for 14 days [1]. This value was used as an estimate of the upper range of plasma glucose fluctuation in the normal rats. None of the insulin-treated diabetic rats whose sciatic nerves were studied had a tail vein plasma glucose concentration that exceeded $152 \mathrm{mg} / \mathrm{dl}$ in any of the twice daily determinations carried out during the 6 th through the 14th days. The mean \pm SEM of the average plasma glucose concentration found in each of the 6 animals in the group during this period was $68 \pm 4 \mathrm{mg} / \mathrm{dl}$.

Five groups of rats were prepared for examination of their sciatic nerves on day 14; each group corresponded to an identical group in whom sciatic MNCV had been determined on day 14 as previously reported [1]. The groups were: (1) normal rats fed the standard diet; (2) diabetic rats fed the standard diet for 14 days following streptozotocin administration; (3) diabetic rats fed the standard diet and treated with insulin from days 3 through 14 , as described above; (4) diabetic rats fed the standard diet supplemented with $1 \%$ free myoinositol for 14 days following streptozotocin administration; (5) normal rats fed the standard diet supplemented with $1 \%$ free myoinositol for 14 days. Plasma glucose was determined in tail vein samples, as previously described [1]. Body weight was determined daily in the diabetics and every other day in the normals.

On day 14 , the rats were anaesthetized with pentobarbital $(0.04 \mathrm{mg} / \mathrm{g}$ wt, IP); the sciatic nerves were exposed surgically from the sciatic notch to the popliteal fossa, quickly excised and minced in $4 \%(\mathrm{~W} / \mathrm{V})$ glutaraldehyde in $0.1 \mathrm{~mol} / \mathrm{l}$ phosphate buffer, $\mathrm{pH} 7.4$, and fixed in the same solution at room temperature for a minimum of 3 hours. The tissue was then immersed in 30\% glycerol in $0.1 \mathrm{~mol} / 1$ phosphate buffer, $\mathrm{pH} 7.4$, for at least 30 minutes, rapidly frozen in Freon 22, cooled in liquid nitrogen, and fractured and shadowed in a Balzers BAF 301 apparatus (Balzers Instruments, Balzers, Liechtenstein), according to the method of Moor and Muhlethaler [10]. The freeze-fracture replicas were cleaned in a sodium hypochlorite solution (47.6 g of active chlorine per litre) for two hours, rinsed in distilled water, mounted on copper grids, and examined in a Philips EM 300 electron microscope (Philips Instruments, Eindhoven, The Netherlands). Magnifications were calibrated with a reference grid (2160 lines $/ \mathrm{mm})$. Thin sections of randomly selected samples from each nerve were also studied; for this purpose, tissue samples fixed in glutaraldehyde were postfixed in $2 \%(\mathrm{~W} / \mathrm{V})$ osmium tetroxide in $0.02 \mathrm{~mol} / \mathrm{l}$ phosphate buffer, $\mathrm{pH} 7.4$, washed with distilled water, dehydrated in graded con- centrations of ethanol, and embedded in Epon. 'Thin sections were stained with uranyl acetate and lead citrate and examined in a Philips EM 300 electron microscope.

The quantitative evaluation of the freeze-fracture replicas was carried out by observers unaware of the identity of the samples being studied. A minimum of two different replicas were randomly selected from the material from each animal, and large myelinated Fibres chosen at random in each replica. Fifteen flat inner faces $(P$ faces) and ten flat outer faces (E-faces) of different internodal membrane segments were photographed. Each membrane face was photographed at two fixed magnifications : $35,000 \times$ for the evaluation of the number of particles and $58,000 \times$ for the determination of their size. Measurements of the number and size of intramembranous particles were performed on positive prints enlarged 3 times (i.e. final magnification $105,000 \times$ and $175,000 \times)$. A rectangular grid calibrated to represent $0.25 \mu \mathrm{m}^{2}$ was superimposed on the centre of the prints, avoiding the circumscribed particle free-patches ${ }^{1}$; the number of particles present within this area were counted, and the results expressed as number of particles per $\mu \mathrm{m}^{2}$. The average particle number per unit area of $\mathrm{P}$-face and of E-face was computed for the material from each animal, and used for the computation of the mean \pm SEM of the group given in Table 1. Moreover, in normal and diabetic animals, frequency histograms were constructed on the basis of the number of intramembranous particles per test-grid in all P-faces (75) and E-faces (50) analysed in these two conditions. The measurements of the size of the intramembranous particles found in the P-faces were performed with the aid of an $8 \times$ magnifier containing a graticule calibrated in tenths of a millimeter. The size of all the particles present within a fixed area $\left(0.25 \mu \mathrm{m}^{2}\right)$ in the centre of the pictures of 10 different $\mathbf{P}$-faces from each animal was determined (representing a total of approximately 2,500 particles per experimental condition). Particles have a round shape and their diameter was measured perpendicularly to shadowing.

Ten pictures chosen at random from all the positive prints (final magnification 105,000 $\times$ ) of P-faces from the nerves of the normal and acute streptozotocin diabetic rats fed the standard diet were subjected to a planimetric analysis of the total area of internodal myelin freeze-fracture surfaces included in the photograph and the total area of any particle-free zones included in the photograph. At the end of the analysis these data were utilized to compute the total area of myelin freeze-fracture surface included in each randomly selected sample, and the fraction of the total area occupied by particle-free zones given in Table 1 .

\section{Results}

The EM appearance of the myelinated nerve fibres in thin sections of the sciatic nerves from the normal controls corresponded in detail to that reported by other workers [11]. There were no qualitative alterations in the EM appearance of the myelinated nerve fibres in thin sections of the sciatic nerves of rats with streptozotocin diabetes of 14 days duration. Similarly

\footnotetext{
1 According to Pinto da Silva \& Miller [9], these particle-free areas represent artefacts which can be avoided by freezing the tissue without fixation and/or exposure to cryoprotectant. In our hands, subsequent fractures of rat sciatic nerves frozen without prior glutaraldehyde fixation and glycerol treatment, yielded such low amount of well preserved myelin fracture faces that this material was not suitable for a quantitative analysis of membrane morphology.
} 


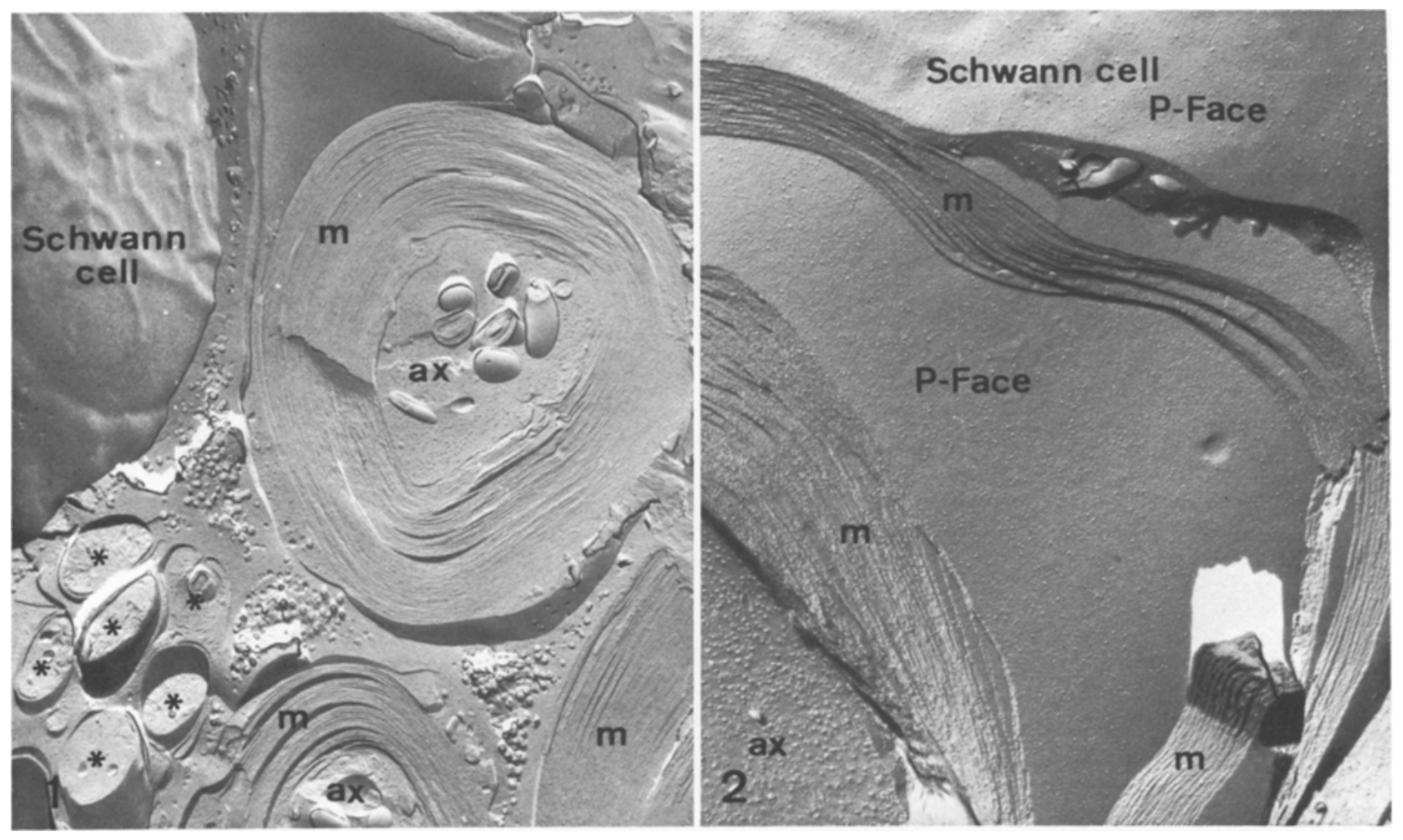

Fig. 1. Electron micrograph of a freeze-fracture replica of a portion of the sciatic nerve of a normal rat showing both myelinated axons (ax) and non-myelinated axons $\left({ }^{*}\right)$ in cross fracture. In this orientation the myelin sheaths $(\mathrm{m})$ surrounding the myelinated axons appear as concentric rings. Part of the membrane of a Schwann cell body is seen in the upper right corner. Magnification $\times 8,000$

Fig. 2. Electron micrograph of a freeze-fracture replica of a portion of a large myelinated sciatic nerve fibre from a normal rat. The fracture extends from the membrane of the Schwann cell body (Schwann cell P-face) to the axoplasm (ax). The fracture through the myelin sheath reveals both longitudinally fractured lamellae (P-face) and cross fractured layers of myelin lamellae (m). Magnification $\times 24,000$

there were no qualitative alterations in the EM appearance of myelinated sciatic nerve fibres in tissue from diabetic rats treated with insulin or fed the diet supplemented with $1 \%$ myoinositol, or from normal rats fed the same diet.

The general appearance of the myelinated nerve fibres in freeze-fracture replicas of sciatic nerves from the normal rats fed the standard diet is illustrated in Figures 1,2, and 3. The replicas of the fractured fibres permit the examination of the membrane face of the myelin sheaths and also of the membrane faces of their corresponding Schwann cell bodies. The myelin sheath is recognized by its characteristic layered appearance, while the Schwann cell is distinguished by its typical location at the external side of the myelin sheath and by the presence of microinvaginations at its membrane surface. There were no significant differences in the qualitative appearance of the myelinated nerve fibres in freeze-fracture replicas of sciatic nerves from the streptozotocin diabetic rats fed the standard diet when compared with those from the normal controls.

Two complementary fracture faces result from splitting the myelin membrane along its hydrophobic matrix. One corresponds to the inner leaflet of the membrane, the P-face, and the other, the E-face to the outer leaflet of the membrane $[8,9,12]$. The typical appearance of the myelin fracture faces is that of smooth surfaces containing globular protrusions, the intramembranous particles, which are thought to represent integral membrane proteins. In the freezefracture surfaces of the internodal myelin of large sciatic nerve fibres of normal and acute streptozotocin diabetic rats these intramembranous particles are uniformly distributed (Fig. 3) with the exception of focal, well circumscribed areas that are free of particles (Fig. 4), when the nerve is fixed in glutaraldehyde and treated with glycerol in the conventional manner prior to freeze-fracture. As detailed in Methods the particle-free areas were excluded from the morphometric analysis. As shown in Table 1, the total area of the particle-free zones varied from 3.5 to $5.6 \%$ of the total area of the internodal myelin included in randomly selected samples from the large sciatic nerve fibres of normal rats fed the standard diet, with a mean of $4.5 \%$. The mean value in randomly selected samples from the acute streptozotocin diabetic rats fed the same diet averaged $3.8 \%$. 


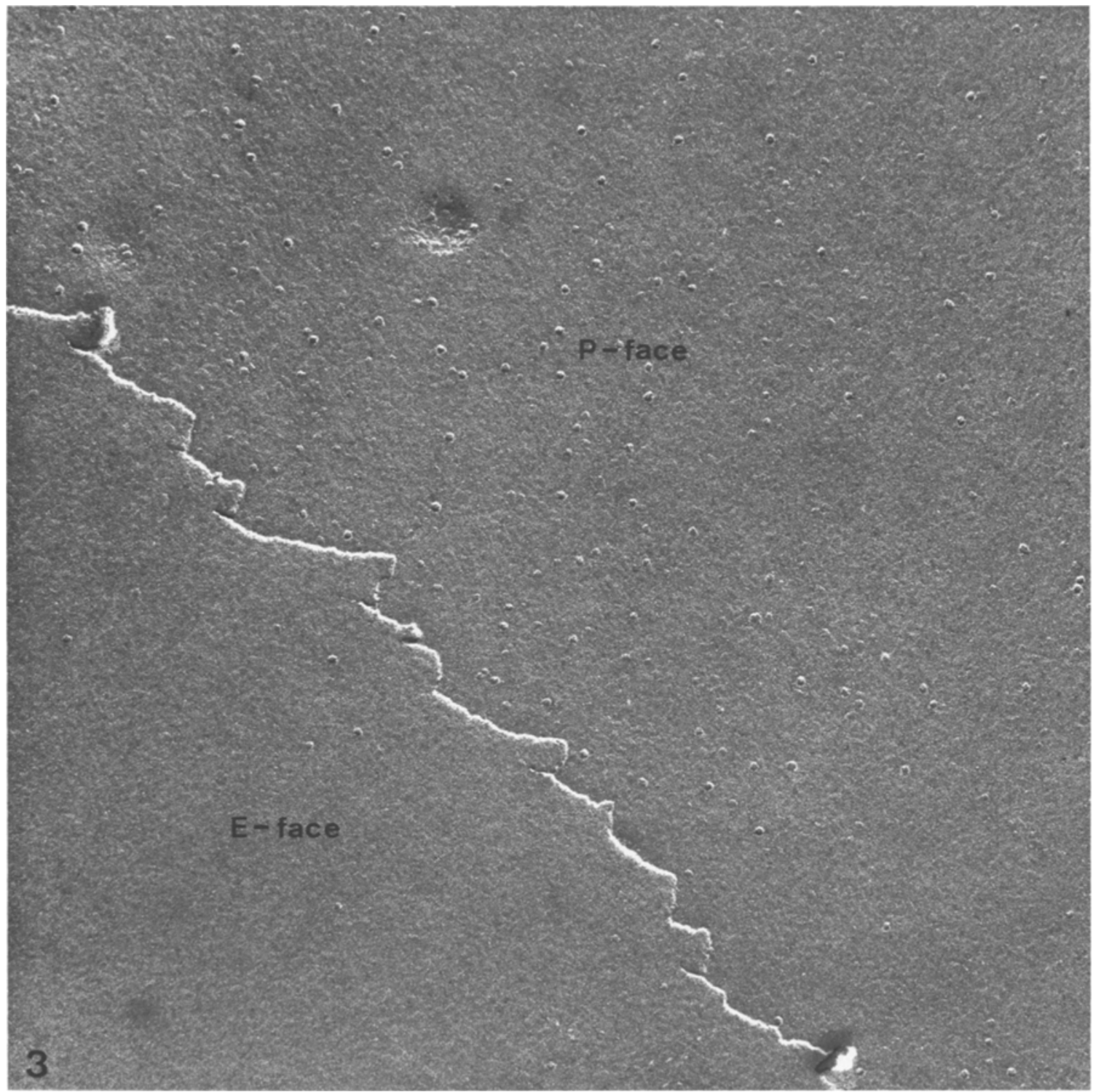

Fig. 3. Detail of an electron micrograph of a freeze-fracture replica of part of the myelin sheath of a large myelinated sciatic nerve fibre from a normal rat. (Magnification $\times 100,000$ ). The P-face and E-face of the freeze-fracture surfaces of adjacent myelin layers are shown. The density of intramembranous particles in the P-face clearly exceeds that in the E-face

Hence, the method of preparation provides reproducibly similar replicas for analysis and comparison in nerves from normal and diabetic rats.

The P-face of the internodal myelin of large sciatic nerve fibres from both normal and acute streptozotocin diabetic rats have a greater density of randomly distributed intramembranous particles than the E-faces in the same sheath.

In the internodal myelin from the normal controls fed the standard diet the average density of intramembranous particles per unit area of P-face and $\mathrm{E}$-face in different animals varied within a relatively narrow range (Table 2). The variation in particle distribution between test grids in each animal in the control group is shown in Figure 5; particle aggregation was not observed.

In the internodal myelin of large sciatic nerve fibres from the streptozotocin diabetic rats fed the standard diet there was a significant decrease in the average density of intramembranous particles per unit area of P-face and E-face (Table 2). These decreases were not associated with any significant increase in the variation in particle distribution between test grids in each diabetic animal (Fig. 5), and particle aggregation was not observed.

Insulin treatment of acute streptozotocin diabetic rats prevented the development of a decrease in the average density of intramembranous particles in the 


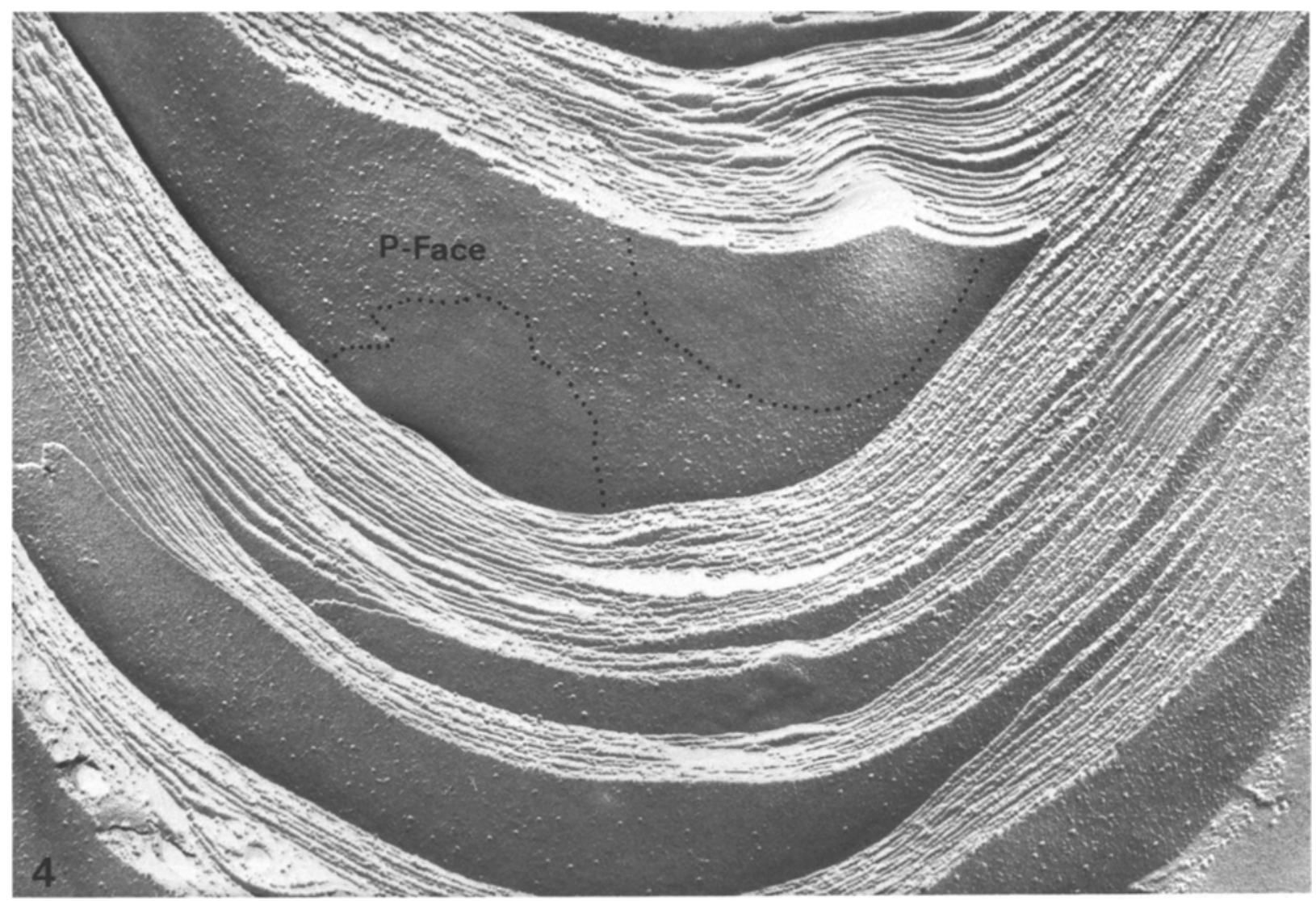

Fig. 4. Electron micrograph of a freeze-fracture replica of part of the myelin sheath of a large myelinated sciatic nerve fibre from a normal rat at a magnification of $\times 49,000$, showing both longitudinally and cross fractured myelin lamellae. On the P-face of the exposed myelin membrane there are well defined areas which are devoid of intramembranous particles (outlined by the dotted lines). The cross fractured myelin sheath also contains well circumscribed areas in which the fracture faces are devoid of particles. The particle-free areas in the longitudinally fractured membrane appear to correspond to particle-free areas in the cross fractured layers

Table 1. Planimetric estimation of the total area of the internodal myelin freeze-fracture surfaces included in 10 randomly selected P-faces from large sciatic nerve fibres and the total area occupied by the well circumscribed particle-free zones. $F=A r e a$ of particle-free zones/ total area

\begin{tabular}{|c|c|c|c|c|c|c|c|}
\hline \multicolumn{4}{|c|}{ Normal rats (Standard diet) } & \multicolumn{4}{|c|}{ Diabetic rats (Standard diet) } \\
\hline & $\begin{array}{l}\text { Total area } \\
\mu \mathrm{m}^{2}\end{array}$ & $\begin{array}{l}\text { Area of } \\
\text { particle-free-zones }\end{array}$ & $\mathrm{F}$ & & $\begin{array}{l}\text { Total area } \\
\mu \mathrm{m}^{2}\end{array}$ & $\begin{array}{l}\text { Area of } \\
\text { particle-free-zones }\end{array}$ & $\mathrm{F}$ \\
\hline Rat 1 & 21.18 & 1.10 & 0.052 & Rat 6 & 19.81 & 0.61 & 0.033 \\
\hline Rat 2 & 26.44 & 1.33 & 0.050 & Rat 7 & 25.31 & 0.94 & 0.037 \\
\hline Rat 3 & 22.72 & 0.79 & 0.035 & Rat 8 & 20.73 & 1.16 & 0.056 \\
\hline Rat 4 & 27.32 & 1.02 & 0.037 & Rat 9 & 18.62 & 1.45 & 0.024 \\
\hline \multirow[t]{2}{*}{ Rat 5} & 26.48 & 1.04 & 0.039 & Rat 10 & 22.80 & 0.88 & 0.039 \\
\hline & & \multicolumn{4}{|c|}{ Mean $\pm \mathrm{SEM}=0.043 \pm 0.004$} & \multicolumn{2}{|c|}{ Mean + SEM $=0.038+0.005$} \\
\hline
\end{tabular}

P-face and E-face of the internodal myelin of large sciatic nerve fibres (Table 2). This alteration was also prevented by feeding the acute streptozotocin diabetic rats the diet supplemented with $1 \%$ free myoinositol (Table 2), although these rats were persistently hyperglycaemic (Table 2).

The size of the intramembranous particles found in the P-face of the internodal myelin of large sciatic nerve fibres in each of the experimental conditions examined is shown in Figure 6. The analysis was performed in the P-face since the number of particles per unit area is 3 times greater than in the E-face, and since the decrease in particle density associated with streptozotocin diabetes was more striking in the 
Table 2. Density of intramembranous particles in the freeze-fracture faces of myelin membranes of large myelinated fibres in the sciatic nerves of normal and acute streptozotocin diabetic rats

\begin{tabular}{|c|c|c|c|c|c|c|c|}
\hline \multirow{3}{*}{ Type of rat } & \multirow{3}{*}{ Diet } & \multirow{3}{*}{$\mathrm{n}^{\mathrm{a}}$} & \multirow{3}{*}{$\begin{array}{l}\text { Plasma glucose } \\
\mathrm{mg} / \mathrm{dl}\end{array}$} & & & \multicolumn{2}{|c|}{$\begin{array}{l}\text { Data from Greene et al. } \\
\text { [1] shown for comparison: }\end{array}$} \\
\hline & & & & \multicolumn{2}{|c|}{ Number of particles per $\mu \mathrm{m}^{2}$ of membrane } & Sciatic MNCV ${ }^{c}$ & $\mathrm{n}$ \\
\hline & & & & P-Face & E-Face & \multicolumn{2}{|l|}{$\mathrm{M} / \mathrm{Sec}$} \\
\hline Normal & Standard & $(5)$ & $144 \pm 11$ & $140.4 \pm 4.7$ & $38.4 \pm 1.8$ & $64.6 \pm 0.9$ & $(20)$ \\
\hline Diabetic & Standard & $(5)$ & $751 \pm 56$ & $123.3 \pm 2.4$ & $32.8 \pm 1.2$ & $50.1 \pm 0.9$ & (35) \\
\hline $\begin{array}{l}\text { Diabetic- } \\
\text { Insulin Rx }\end{array}$ & Standard & (6) & $72 \pm 17$ & $138.4 \pm 5.5$ & $39.3 \pm 1.8$ & $62.9 \pm 0.9$ & $(17)$ \\
\hline Diabetic & $+1 \% \mathrm{MI}^{\mathrm{d}}$ & $(6)$ & $733 \pm 26$ & $141.1 \pm 4.7$ & $38.6 \pm 1.7$ & $58.0 \pm 1.4$ & (19) \\
\hline Normal & $+1 \% \mathrm{MI}$ & (6) & $141 \pm 22$ & $138.6 \pm 1.2$ & $38.4 \pm 1.9$ & $64.2 \pm 1.4$ & (14) \\
\hline
\end{tabular}

All values (obtained on Day 14) are expressed as mean \pm SEM

a $n=$ number of animals studied

b Statistical significance was determined with Student's t-test

c $\mathrm{MNCV}=$ Motor nerve conduction velocity

d $\mathrm{MI}=$ Myoinositol
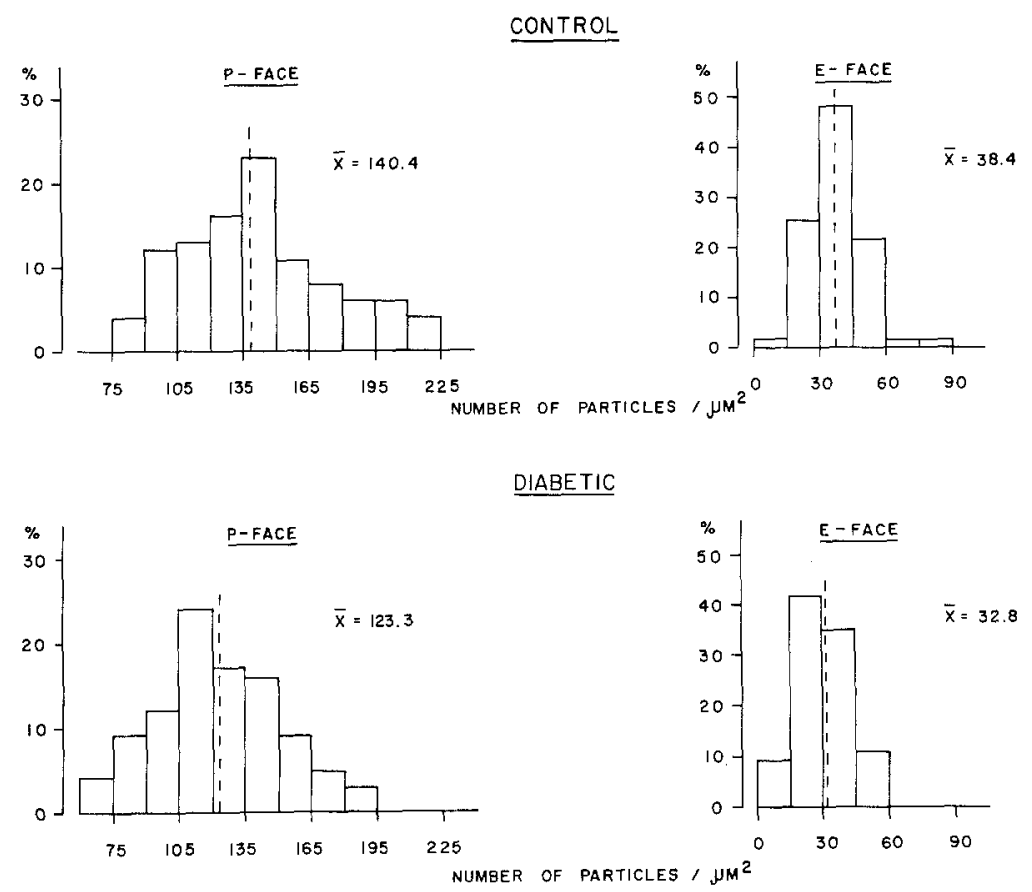

CONTROL

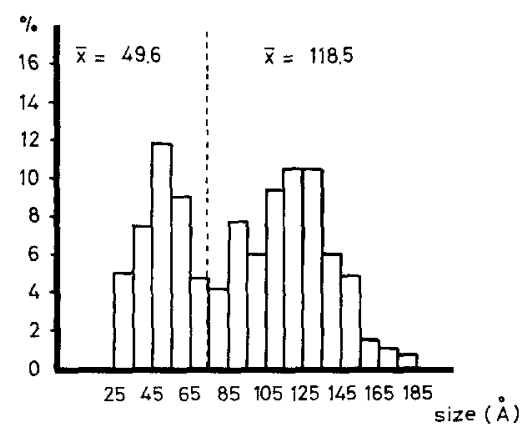

DIABETIC

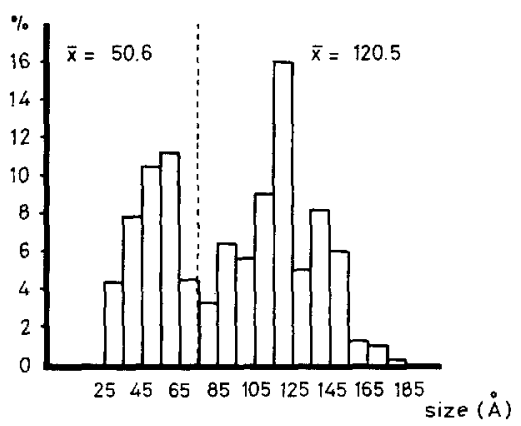

Fig. 5. Frequency histogram of the number of particles per test grid analysed in myelin membranes of control and diabetic rats. Pand E-face particles show a symmetrical frequency around the mean $(\bar{x})$ in both conditions analysed, thus excluding a clustering of particles as the cause of the different numbers of particles counted in diabetic and control rats

Fig. 6. Distribution of the sizes (diameter in $\AA$ ) of the particles found in the P-freezefracture surface of the internodal myelin of large sciatic nerve fibres from control and acute streptozotocin diabetic rats. The data for each of the experimental conditions indicated were derived from the measurement of approximately 2500 particles. The mean size $(\tilde{\mathbf{x}})$ for two groups of particles (diameters less than $75 \AA$ and greater than $75 \AA$ ) are given for each of the experimental conditions 
P-face. In the P-face of the internodal myelin of large sciatic nerve fibres from the normal controls, the size of the intramembranous particles had a bimodal distribution with one class of particles ranging from 25 to $75 \AA$ in diameter with a mean of $49.6 \AA$ and a second group that ranged from 75 to $185 \AA$ in diameter with a mean of $118.5 \AA$ (Fig. 6). The distribution of intramembranous particle sizes, and the mean diameters of the two classes of particles were not significantly altered in the P-face of the internodal myelin of large sciatic nerve fibres from the streptozotocin diabetic rats fed the standard diet (Fig. 6).

\section{Discussion}

Electron microscopic examination of the freeze-fracture faces of the internodal myelin of peripheral nerves in several species [9] suggests that its structure conforms to the fluid mosaic model [13] in which globular molecules of integral membrane proteins alternate with sections of phospholipid bilayer. The intramembranous particles present in the freeze-fracture faces of the internodal myelin of rat large sciatic nerve fibres are thus believed to represent integral membrane proteins. The structural and functional significance of these particles in the myelin membrane remains to be determined, but it has been suggested that some may be important in the maintenance of the structural and organizational integrity of the myelin sheath [9].

The observations reported above demonstrate that acute experimental diabetes results in a significant decrease in the average distribution density of intramembranous particles in both the P-and E-faces of the internodal myelin membrane of large sciatic nerve fibres. This decrease does not appear to result from particle aggregation, from a change in the random distribution of intramembranous particles, or from the selective loss of particles of a specific size. These observations suggest that the internodal myelin membrane of large sciatic nerve fibres in rats with acute streptozotocin diabetes has a decreased number of integral protein particles per unit area, which would constitute a significant alteration in the structure of this membrane. This alteration is clearly a consequence of insulin deficiency and/or hyperglycaemia since it is prevented by a rigorous insulin treatment that obviates hyperglycaemia from days 6 through 14 after the induction of diabetes. The myelin membrane is derived from the plasma membrane of the Schwann cell, but its composition is significantly modified in the course of the formation of the myelin sheath. There were no qualitative alterations in the EM appearance in freeze-fracture replicas. Since the data presently available on the metabolism of the Schwann cells and their metabolic interactions with the axons are fragmentary there is no firm basis for efforts to localize the site of the metabolic derangements that may be responsible for the alteration in the structure of the internodal myelin membrane. Although Spritz et al. [14] found no difference in the protein content of myelin isolated and purified from the peripheral nerves of normal and acute streptozotocin diabetic rats or in their pattern on polyacrylamide disc-gel electrophoresis, the methodology employed would not preclude the existence of an alteration in the structure of internodal myelin in the diabetic. The same workers reported that acute streptozotocin diabetes results in a 30 to $80 \%$ decrease in the incorporation of DL-(1${ }^{14} \mathrm{C}$ ) leucine into myelin protein by segments of rat sciatic nerve incubated in vitro [14], and suggested that insulin deficiency alters some aspects of myelin metabolism.

The alteration in internodal myelin membrane structure in rats with acute experimental diabetes was also prevented by a diet containing $1 \%$ free myoinositol despite the persistence of marked hyperglycaemia. Acute experimental diabetes results in a significant decrease in the free myoinositol content of whole sciatic nerve [1], and Hawthorne and his co-workers [15] have recently demonstrated that this is associated with a decrease in whole nerve phosphatidyl inositol content. Phosphatidyl inositol is the precursor of the polyphosphoinositides which are known to be present in very high concentrations in central nervous system myelin [16], and whole peripheral nerve [17]. At present an accurate assessment of the effects of acute experimental diabetes and any form of treatment on the concentrations of specific inositol containing phospholipids in purified peripheral nerve myelin is precluded by the marked lability of the polyphosphoinositides under the conditions required to purify myelin from whole nerve [18]. The diet containing $1 \%$ myoinositol maintains whole sciatic nerve free myoinositol concentrations in the diabetic rats similar to those found in normal rats fed the same diet [1], and does not induce any detectable alteration in the number of intramembranous particles per unit area of the freeze-fracture surfaces of the internodal myelin in normal rats. Since insulin treatment also obviates any decrease in nerve free myoinositol content in acute streptozotocin diabetic rats fed a standard diet containing $0.011 \%$ myoinositol it is reasonable to speculate that the effects of acute experimental diabetes on the structure of internodal myelin in large sciatic nerve fibres is mediated in part through alterations in nerve myoinositol metabolism.

The design of this study required that both sciatic 
nerves be used for morphological examination; this precluded determinations of sciatic $\mathrm{MNCV}$, since the manipulations and electrical stimulation required for such determinations might induce morphological artefacts. However, each of the groups whose nerves were studied morphologically corresponded in detail to a group included in a previously reported extensive study of the effects of acute experimental diabetes, insulin treatment, and dietary myoinositol content on sciatic MNCV in rats [1]; the observations included in that report have been repeatedly confirmed in this laboratory during the period in which the animals used for these morphological studies were prepared; thus a comparison of the morphological observations and the previously reported sciatic MNCV's is appropriate. This comparison (Table 2) suggests a possible relationship between the alteration in internodal myelin structure in rats with acute streptozotocin diabetes and the decreased sciatic MNCV present in such animals. The alteration in internodal myelin structure develops within the same time period as the decrease in sciatic $\mathrm{MNCV}$, and the decrease in the electrical resistance of internodal myelin in rat large sciatic nerve fibres reported by Eliasson [4]. Moreover, insulin treatment by the regimen that consistently prevents the development of the structural alteration in internodal myelin prevents the development of impaired sciatic MNCV. Similarly a diet containing $1 \%$ myoinositol prevents the development of the structural alteration in the internodal myelin and also prevents the development of a significant impairment in sciatic MNCV. These observations suggest that acute experimental diabetes induces an alteration in the structure of the myelin membrane in the internodal myelin of large sciatic nerve fibres, which may contribute to the decreased electrical resistance of the internodal myelin and the decreased sciatic MNCV. This could provide an explanation for the development of decreased sciatic MNCV in the absence of EM evidence of segmental demyelination, myelin oedema, or the loss of large myelinated nerve fibres. This speculation merits detailed evaluation. To date there have been no systematic comparisons of the physical characteristics of internodal myelin in the peripheral nerves of normal and acute experimentally diabetic rats, employing methods such as $\mathrm{x}$-ray diffraction which might detect alterations in the organization of the myelin sheath not apparent on EM pictures.

Acknowledgements. This work was supported in part by research grants 3.553.75 and 3.120.77 from the Swiss National Science Foundation and AM-04722 from the National Institutes of Health.

The authors wish to thank Mrs. Sarah Aquino and Marthe Sidler, Misses Irina Barinov and Isabelle Bernard, and Mr. Patrice Fruleux for their expert assistance.

\section{References}

1. Greene, D. A., DeJesus, Jr., P. V., Winegrad, A. I.: Effects of insulin and dietary myoinositol on impaired peripheral motor nerve conduction velocity in acute streptozotocin diabetes. J. Clin. Invest. 55, 1326-1336 (1975)

2. Eliasson, S. G.: Nerve conduction changes in experimental diabetes. J. Clin. Invest. 43, 2353-2358 (1964)

3. Gregersen, G.: Diabetic neuropathy: influence of age, sex, metabolic control, and duration of diabetes on motor conduction velocity. Neurology (Minneap.) 17, 972-980 (1967)

4. Eliasson, S. G.: Properties of isolated nerve fibres from alloxanized rats. J. Neurol. Neurosurg. Physchiatry 32, 525-529 (1969)

5. Sharma, A. K., Thomas, P. K.: Peripheral nerve structure and function in experimental diabetes. J. Neurol. Sci. 23, 1-15 (1974)

6. Jakobsen, J.: Axonal dwindling in early experimental diabetes. II. A study of isolated nerve fibres. Diabetologia 12, 547-553 (1976)

7. Sharma, A. K., Thomas, P. K., De Molina, A. F.: Peripheral nerve fibre size in experimental diabetes. Diabetes 26, 689-692 (1977)

8. Livingston, R. B., Pfenninger, K., Moor, H., Akert, K.: Specialized paranodal and interparanodal glial-axonal junctions in the peripheral and central nervous system: a freezeetching study. Brain Res. 58, 1-24 (1973)

9. Pinto da Silva, D., Miller, R. G.: Membrane particles on fracture faces of frozen myelin. Proc. Natl. Acad. Sci. USA 72, 4046-4050 (1975)

10. Moor, H., Muhlethaler, K.: Fine structure in frozen etched yeast cells. J. Cell Biol. 17, 609-628 (1963)

11. Wood, J. G., Engel, E. L.: Peripheral nerve glycoproteins and myelin fine structure during development of rat sciatic nerve. J. Neurocytol. 5, 605-615 (1976)

12. Branton, D., Bullivant, S., Gilula, N. B., Karnovsky, M. J., Moor, H., Muhlethaler, K., Northcote, D. H., Packer, L., Satir, B., Satir, P., Speth, V., Staehelin, L. A., Steere, R. L., Weinstein, R.S.: Freeze-etching nomenclature. Science 190, 54-56 (1975)

13. Singer, S. J., Nicolson, G. L.: The fluid mosaic model of the structure of cell membranes. Science 175, 720-730 (1972)

14. Spritz, N., Singh, H., Marman, B.: Metabolism of peripheral nerve myelin in experimental diabetes. J. Clin. Invest. 55, 1049-1056 (1975)

15. Palmano, K.P., Whiting, P.H., Hawthorne, J. N.: Free and lipid myoinositol in tissues from rats with acute and less severe streptozotocin-induced diabetes. Biochem. J. 167, 229-235 (1977)

16. Hauser, G., Eichberg, J., Gonzalez-Sastre, F.: Regional distribution of polyphosphoinositides in rat brain. Biochim. Biophys. Acta 248, 87-95 (1971)

17. Sheltawy, A., Dawson, R. M.C.: The polyphosphoinositides and other lipids of peripheral nerves. Biochem. J. 100, 12-18 (1966)

18. Hauser, G., Eichberg, J.: Improved conditions for the preservation and extraction of polyphosphoinositides. Biochim. Biophys. Acta 326, 201-209 (1973)

Received: September 23, 1977,

and in revised form: February 27, 1978

Dr. L. Orci

Institut d'Histologie et d'Embryologie

Ecole de Médecine

CH-1211 Genève 4

Switzerland 